\title{
TAGUNG
}

\section{Europäische Gesundheitspolitik - ein echter Mehrwert für die Bürgerinnen und Bürger?!}

\author{
Ulla Kalbfleisch-Kottsieper*
}

In Weimar trafen sich Vertreter verschiedener Fachrichtungen $\mathrm{zu}$ einem interdisziplinären Erfahrungsaustausch zur Frage, ob die europäische Gesundheitspolitik auch ohne echte, formalrechtliche Kompetenzen ,bürgernah und effizient" sein könne. An der Diskussion beteiligten sich Vertreter der Rechts- und Politikwissenschaft, der Psychologie und Medizin sowie Praktiker aus der Ministerialbürokratie, der Landtagsverwaltung, aus dem Bereich der Gewerkschaften, Krankenkassen und des Verbraucherschutzes.

Vor dem Hintergrund der gescheiterten Ratifizierung einer europäischen Verfassung gewinnt die Auseinandersetzung mit den sogenannten eigentlichen ,Politiken" der Europäischen Union beziehungsweise der Europäischen Gemeinschaften besondere Bedeutung. Diese betreffen die europäischen Bürgerinnen und Bürger oft viel unmittelbarer als solch ,symbolische" Akte wie die Verabschiedung einer Verfassung oder auch Neukonstruktion von Institutionen und Verfahren der Entscheidungsfindung in der Europäischen Union. Dies gilt in besonderem Maße für die Gesundheitspolitik, deren bisherige formale Verankerung im Vertrag zur Gründung der Europäischen Gemeinschaft (Artikel 3, 1p und Artikel 152 EGV) eher schwach entwickelt ist. Der Europäischen Kommission ist es dennoch in den letzten Jahren gelungen, auch spektakuläre Erfolge zu erzielen - die Durchsetzung des Nichtraucherschutzes ist dafür nur ein aktuelles Beispiel. Ihre Beharrlichkeit, dieses Thema über Jahre hinweg immer wieder - und letztlich auch unter Mitwirkung der Mitgliedstaaten - auf die Agenda

\section{Die Europäische Gesundheitspolitik: bürgernah und effizient auch ohne Kompetenzen?}

Wissenschaftliche Tagung des Arbeitskreises Europäische Integration und des Instituts für Europäische Politik mit freundlicher Unterstützung der Europäischen Kommission und der ASKO-Europa-Stiftung

Weimar, 1./2. Juni 2007

Wissenschaftliche Leitung:

Ulla KALBFLEISCH-KOTTSIEPER, Institut für Europäische Politik, Berlin

Begrüßung und Einführung

Ulla KALBFLEISCH-KOTTSIEPER, Institut für Europäische Politik, Berlin

Die sozialrechtliche Dimension der europäischen Gesundheitspolitik

Prof. Dr. Dr. Eberhard EICHENHOFER, Friedrich Schiller Universität, Jena

Deutsche Interessen an einer europäischen Gesundheitspolitik

Ortwin SCHULTE, Bundesministerium für Gesundheit, Berlin

Paneldiskussion: Fragen aus der Praxis: Welchen europäischen Regelungsbedarf sehen Ärzte, Patienten, Krankenkassen und andere stakeholder der Gesundheitswirtschaft?

PD Dr. Stefan SCHRÖDER, Chefarzt der Klinik für Psychiatrie und Psychotherapie KMG-Kliniken Güstrow

Guido DRESSEL, Leiter der Techniker Krankenkasse, Thüringen

Dr. Ralph WALTHER, Verbraucherzentrale Thüringen, Erfurt

Moderation: Ulla KALBFLEISCH-KOTTSIEPER, Institut für Europäische Politik, Berlin

\footnotetext{
* Ulla Kalbfleisch-Kottsieper, Ministerialdirigentin, Institut für Europäische Politik, Berlin.
} 
gesetzt und seine politische Umsetzung vorangetrieben zu haben, macht zum einen den ,europäischen Mehrwert' gemeinschaftlichen Handelns deutlich. Zum anderen zeigt es aber auch, dass es nicht unbedingt traditioneller Kompetenzübertragung durch konstitutionelle Akte bedarf, um europäischen Fortschritt zu erreichen.

Europäische Gesundheitspolitik im sozialrechtlichen Kontext

In seinem Einführungsvortrag wies Eberhard Eichenhofer darauf hin, dass entgegen der in der Politik gerne verbreiteten Auffassung, Sozialpolitik sei im Wesentlichen immer noch nationale Aufgabe, dies von der europäischen Realität längst überholt sei. So sehe auch der Entwurf des Vertrags über eine Verfassung für Europa (VVE) sowohl eine offiziell ,geteilte Zuständigkeit“" (Artikel I-14 VVE) als auch eine sogenannte „Sozialklausel“ (Artikel III-117 VVE) vor. In Artikel III-136 VVE sei sogar eine „kleine europäische Kompetenz“ in Richtung auf die sozialen Sicherungssysteme enthalten, gemäß der zu- und abwandernden Arbeitnehmern im europäischen Binnenmarkt die ,Zusammenrechnung aller nach den verschiedenen innerstaatlichen Rechtsvorschriften zu berücksichtigenden Zeiten für den Erwerb und die Aufrechterhaltung des Leistungsanspruchs sowie für die Berechnung der Leistungen" garantiert werden solle. Als ,Notbremse' für die Nationalstaaten - und auch zur Wahrung des Subsidiaritätsprinzips - sei vorgesehen gewesen, in Zweifelsfällen den Europäische Rat mit dem entsprechenden Entwurf eines Europäischen Gesetzes beziehungsweise Rahmengesetzes zu befassen. Eichenhofer ging ebenfalls davon aus, dass die europäische Integration, seit einem halben Jahrhundert ein wirtschaftliches Vorhaben, inzwischen kaum merklich, jedoch ausweislich ihrer Rechtsordnung auch zu einem sozialpolitischen Projekt geworden sei. Die Mitgliedstaaten der Europäischen Union seien „Zeugen und Beteiligte einer Europäisierung des sozialen Schutzes“. Im Übrigen sei der
EU-Gesundheitspolitik im Praxis-Check: Die Europäische Versicherungskarte - ein bestpractice Beispiel?

Dr. Gottfried DIETZEL, e-health-Consultancy, Bonn

Position des DGB zur Mitteilung der Kommission über Gemeinschaftsmaßnahmen im Bereich der Gesundheitsdienstleistungen Inge KAUFMANN, Deutscher Gewerkschaftsbund, Berlin/Brüssel

Gesundheitspolitische Interessen im Entwurf der Europäischen Verfassung

Dr. Martin BOROWSKY, Richter am Landgericht Erfurt

EU-Gesundheitspolitik in der regionalen Verantwortung - ein Überblick zur europäischen Kompetenzlage im Bereich der Gesundheitspolitik

Ulla KALBFLEISCH-KOTTSIEPER, Institut für Europäische Politik, Berlin

Schlusspanel: Gute Genesung Europa - EUGesundheitspolitik als Beispiel für ,kompetenzlose" europäische Eigendynamik? Cui bono?

Europäische Binnenmarkt ohne flankierende europäische Sozialpolitik gar nicht denkbar.

Die offene Methode der Koordinierung (OMK) habe sich seit den 1990er Jahren zu einem gut funktionierenden Steuerungsinstrument in der Gesundheits- und Sozialpolitik entwickelt. Dabei sei, wie gelegentlich behauptet wird, in den Mitgliedstaaten kein ,race to the bottom' entstanden, sondern dieser ,Best Practice'-Ansatz habe vielmehr in der Europäischen Union mit ihren transnationalen Wirtschaftsräumen zu einem erheblichen Modernisierungsschub geführt.

\section{Europäische Gesundheitspolitik während der} deutschen Ratspräsidentschaft

Die Sicht des Praktikers und Mitgestalters der deutschen Ratspräsidentschaft der Europäischen Union brachte Ortwin Schulte mit seinem aktuellen Überblick zu den Interessen und Prioritäten der deutschen Regierung auf 
dem Gebiet einer europäischen Gesundheitspolitik ein. Nach wie vor gehe die Bundesregierung prinzipiell vom Vorrang einer nationalstaatlichen Gestaltung der Gesundheitssysteme aus, dennoch sei sie - auch angesichts einer in den letzten Jahren zunehmend an Bedeutung gewinnenden europäischen $\mathrm{Ge}$ sundheitspolitik - zu einem maßvollen Ausbau der Kompetenzgrundlagen in Artikel 152 EGV bereit. Man setze sich für eine stärkere Verbindlichkeit der Zusammenarbeit im Rahmen der offenen Methode der Koordinierung ein, sowie dafür, dass Mittel der Struktur- und Kohäsionsfonds stärker auch auf Gesundheitsprojekte fokussiert werden.

Erstmals wurde eine Ratspräsidentschaft als sogenannte Triopräsidentschaft ${ }^{1}$ konzipiert, was zwar eine intensivere Koordinationsarbeit bedeutet, zugleich jedoch den Vorteil einer insgesamt nachhaltigeren Planung mit sich gebracht habe. Der Aktionsrahmen der deutschen Ratspräsidentschaft sei laut Schulte zunächst einmal von den ohnehin anstehenden legislativen und nicht-legislativen Projekten der Europäischen Kommission bestimmt worden. Dies waren im ersten Halbjahr 2007 unter anderem Vorschläge für Entwürfe zu Verordnungen über Arzneimittel für neuartige Therapien, Richtlinien zu Medizinprodukten, Entwicklung eines Gemeinschaftsrahmens für sichere und hochwertige Gesundheitsdienste, das Grünbuch zum Nichtraucherschutz und vor allem das Zweite Aktionsprogramm der Gemeinschaft im Bereich der Gesundheit (2007-2013) sowie die hierzu vorliegenden Änderungsvorschläge des Europäischen Parlamentes.

Die eigenen inhaltlichen Akzente der deutschen Präsidentschaft in der Gesundheitspolitik könnten mit den drei Leitbegriffen Innovation, Prävention und Zugang zu Gesundheitsdienstleistungen zusammengefasst werden. Innovation meine hier vor allem die Sicherung eines zukunftsorientierten Pharma- standortes in Europa, durch die Förderung von wettbewerbsfähigen Schlüsselbereichen wie zum Beispiel Gentherapie oder Zell- und Gewebeersatz. Prävention betreffe sowohl den Bereich der HIV/AIDS-Bekämpfung und hier vor allem die Einbeziehung einer aktiven Zivilgesellschaft - als auch die Entwicklung einer gemeinschaftlichen Strategie der Mitgliedstaaten gegenüber Krankheiten, die durch Fehlernährung und mangelnde Bewegung verursacht werden. In beiden Bereichen steige die Zahl der Betroffenen ständig, wobei mit diesen Erkrankungen nicht nur erhebliche Einschränkungen individueller Lebensqualität, sondern nicht zuletzt auch erhebliche volkswirtschaftliche Kosten verbunden seien. Beim Zugang zu den Gesundheitsdienstleistungen stehe unter anderem die sich verstärkende grenzüberschreitende Nutzung von Gesundheitsangeboten im Vordergrund - auch wenn derzeit nach Aussage von Schulte lediglich ein Prozent der gesetzlich vorgeschriebenen Krankenversicherungsleistungen Auslandsbehandlungen beträfen. Ziel sei es, einen gemeinschaftlichen Rechtsrahmen zu entwickeln, der zum einen Rechtssicherheit für die Patienten und die Leistungserbringer bietet, gleichzeitig die nationale Verantwortung für die Sozialversicherung und ihre Steuerungsinstrumente wahrt und die Rechtsfortentwicklung nicht dem Europäischen Gerichtshof überlässt. Die bereits 2006 vom Europäischen Rat beschlossenen Grundwerte „Universalität, Zugang zu einer qualitativ guten Gesundheitsversorgung, Gleichbehandlung und Solidarität" sollen dabei Berücksichtigung finden. Erneuter Regelungsbedarf sei auch deshalb entstanden, weil das Europäische Parlament in der zurückliegenden Debatte um die Dienstleistungsrichtlinie auf die Ausklammerung der Gesundheitsdienstleistungen bestanden hatte.

In Hinblick auf die Diskussion um die offene Methode der Koordinierung, die erst seit 2006 auch für die Gesundheitspolitik verstärkt An-

1 Unter anderem wurde mit den in der Ratspräsidentschaft nachfolgenden Mitgliedstaaten Portugal und Slowenien ein gemeinsames 18-Monatsprogramm erstellt. 
wendung finde, nachdem sie zuvor schon in den Bereichen Rente, soziale Ausgrenzung und Beschäftigung eingesetzt worden war, forderte Schulte eine differenzierte Betrachtungsweise: Einigkeit bestehe hinsichtlich des Erfahrungsaustausches und ,Best Practice'. Nicht unumstritten sei hingegen der Einsatz dieser Methode als indikatorengestütztes Monitoring der Reformprozesse in einzelnen Mitgliedstaaten. Grundsätzlich abgelehnt wird seitens der Bundesregierung die juristische Verbindlichkeit dieser Vorgehensweise.

\section{Europäische Gesundheitspolitik im Verfas- sungsvertrag}

Die primärrechtlichen Änderungen im Bereich der Gesundheitspolitik durch eine europäische Verfassung skizzierte Martin Borowsky in seinem Beitrag. Als Koautor eines umfassenden Kommentars zur Charta der Grundrechte der Europäischen Union konnte er aus der ganzen Fülle seiner Erfahrung berichten und bestätigen, dass die Heterogenität der gesundheitspolitischen Vorstellungen der Mitgliedstaaten innerhalb der Europäischen Union doch erheblich seien, was durch die Erweiterung der Europäischen Union um die zehn mittel- und osteuropäischen Staaten noch verstärkt worden sei. Im Mandat des EU-Gipfels von Laeken für den ,Konvent zur Zukunft Europas' sei die Sozialpolitik - und damit auch die Gesundheitspolitik - kaum beachtet worden. Wenn man sich auf eine „Spurensuche" begebe, sei allerdings festzustellen, dass die Grundrechtecharta eine Reihe von gesundheitspolitisch relevanten Artikeln enthalte, wobei das Spektrum vom Recht auf Leben und Unversehrtheit über besondere arbeitsschutzrechtliche Formulierungen bis hin zu Artikel II-95 VVE, der sich speziell mit dem Gesundheitsschutz befasst, reicht. An der Formulierung des Artikels II-95 VVE lasse sich die Ambivalenz der Mitgliedstaaten deutlich ablesen, denn das „Gewähren“ eines Zugangs zur Gesundheitsvorsorge und ärztli- cher Versorgung gelte nur ,nach Maßgabe der einzelstaatlichen Rechtsvorschriften und Gepflogenheiten" - dies könne kaum als wirklich „europäischer Zugewinn“ bezeichnet werden. Im Gesamtentwurf des Verfassungsvertrages findet sich die Gesundheitspolitik unter anderem in den Artikeln über die geteilten Zuständigkeiten (Artikel I-14 VVE), die soziale Sicherheit und soziale Unterstützung, den Gesundheitsschutz (Artikel I-94/95 VVE) sowie zur öffentlichen Gesundheit (Artikel III-278 VVE) wieder, wobei noch unklar sei ob und wie der dritte Teil im Reformvertrag enthalten sein werde. ${ }^{2}$ Borowsky stellte auch fest, dass die europarechtliche Realität in der Europäischen Union hinter den - zumindest in Umfragen - geäußerten, politischen Erwartungen der Bürgerinnen und Bürger zurückbleibe.

\section{Europäische Gesundheitspolitik in der Pra- xis: e-Health}

Enttäuschte Erwartungen gab es bislang auch bei einer scheinbar so simplen und notwendigen Sache wie der europäischen Krankenversicherungskarte - dies wurde im Vortrag von Gottfried Dietzel deutlich, der sowohl als Mitarbeiter der Europäischen Kommission als auch im Bundesgesundheitsministerium wesentlich an der Entwicklung der rechtlichen und technischen Grundlagen zur Einführung dieser Versicherungskarte als auch an der Implementierung der sogenannten e-Health Politik der Europäischen Union beteiligt war. Für Dietzel stellt die elektronische Gesundheitskarte ein (vor allem auch national notwendiges) Instrument und Symbol zur Modernisierung des Gesundheitswesens dar. Genau aus diesem Grund resultierten seiner Meinung nach auch die erheblichen Widerstände in den Reihen sowohl der Ärzteschaft als auch der Krankenkassen, die sich einerseits mit der Einführung der notwendigen neuen Technologien überfordert sähen, ${ }^{3}$ andererseits zum Teil jedoch auch kein Interesse an der hierdurch

2 Im Vertrag von Lissabon finden sich die wesentlichen Artikel zur Gesundheitspolitik in Art. 4 Abs. 2 k, Art. 9 und im Titel XIV (Art. 168) des Vertrags über die Arbeitsweise der Europäischen Union. 
transparenteren Abrechnungspraxis hätten. Volks- und betriebswirtschaftliche Vorteile seien allerdings kaum von der Hand zu weisen, wenn man berücksichtige, dass 20 bis 40 Prozent der Leistungen im Gesundheitswesen Datenerfassungs- und Kommunikationsdienste betreffen und durch effizientere Organisation ein Einsparpotenzial von 35 Milliarden Euro jährlich realisiert werden könnte. Alleine das e-Rezept reduziere die Bearbeitungskosten je Rezept von 0,34 auf 0,07 Euro, was bei rund 750 Millionen Verordnungen jährlich eine Einsparung von 183 Millionen Euro bedeuten würde. Für den im Europäischen Binnenmarkt mobiler gewordenen europäischen Patienten wäre die e-technisch transportable und damit grenzenlos verfügbare Maximalinformation auf der elektronischen Gesundheitskarte eine optimale Verbesserung. Es bedürfe aber noch immer einer erheblichen Akzeptanzoffensive bei den Stakeholdern des Gesundheitssystems, für die das traditionelle Gestrüpp veralteter Kommunikationsformen auch Gerüst tradierter Gewohnheiten sei.

Europäische Gesundheitspolitik aus gewerkschaftlicher Sicht

Um Verständnis für die gewerkschaftliche Position des Verbleibs der Gesundheitspolitik in möglichst umfassender nationaler Verantwortung warb Inge Kaufmann. Es gehe hierbei vor allem auch um politisch gewachsenes Vertrauen. Sie erläuterte nochmals detailliert die Position der Gewerkschaften, die im Zusammenspiel mit dem Europäischen Parlament zur Herausnahme des Gesundheitsbereiches aus der Dienstleistungsrichtlinie geführt habe. Hinsichtlich der Position des Deutschen Gewerkschaftsbundes zur offenen Methode der Koordinierung stellte sie fest, dass sich dieser Ansatz im Bereich des Sozialschutzes durchaus bewährt habe. In der Europäischen Union müsse eine aktive Sozialpolitik verstärkt gefördert werden, jedoch nicht eine eu- ropäische Sozialversicherungspolitik. Auch wenn die Europäische Union global gesehen Modellcharakter hinsichtlich der in ihr insgesamt repräsentierten sozialstaatlichen Grundwerte habe, so könne es jedoch nicht das Ziel sein, ein einziges europäisches Sozialmodell zu entwickeln. Sinnvoll sei es nach Auffassung von Kaufmann aber - gerade im Bereich der Gesundheitspolitik -, die Methode des ,Benchmarking‘ anzuwenden, Mindeststandards - auch im Bereich der Beschäftigungspolitik - zu definieren und durch Aktionsprogramme und Leitlinien, Anregungen für eine insgesamt zukunftsorientierte Gesundheitspolitik in Europa zu geben.

\section{Europäische Gesundheitspolitik im ,Praxis- test}

Die Teilnehmer der Paneldiskussion, Stefan Schröder, Guido Dressel und Ralph Walther brachten die Sicht der sogenannten Stakeholder aus der Praxis in die Debatte ein. Schröder legte besonderen Wert darauf, aus europäischer Sicht immer auch eine globale Verantwortung im Blick zu haben und gerade auch bei der Gesundheitspolitik nicht eine ,nationale Hurrapolitik" zu betreiben. Deutschland liege zwar europaweit an zweiter Stelle was die Ausgaben für das Gesundheitssystem betreffe, ein entsprechender Effizienzgewinn sei aber deshalb nicht unbedingt zu verzeichnen. Zumindest schlage sich dieser nicht in einer höheren Lebenserwartung beziehungsweise auch Lebensqualität im Alter nieder. Einer nicht unumstrittenen - Untersuchung der Weltgesundheitsorganisation (WHO) aus dem Jahr 2000 zufolge, liege Deutschland hinsichtlich der Qualität seines Gesundheitssystems lediglich auf Platz 25 ( bei 191 untersuchten Ländern). Walther stellte zum einen die Arbeit der Patientenbetreuung, einer neuen Aufgabe der Verbraucherzentralen vor, wies aber auch darauf hin, dass die grenzüberschreitende Gesundheitsversorgung - jedenfalls in seinem regionalen Verantwortungsbe-

3 Anfang Oktober 2007 haben die gesetzlichen Krankenkassen mitgeteilt, dass sie sich erst 2009 in der Lage sähen, eine elektronische Gesundheitskarte einzuführen. 
reich - noch keine wesentliche Rolle spiele. Auch Dressel bestätigte, dass sich dieser Posten in den Leistungen der Krankenkassen erst zu maximal einem Prozent der Gesamtausgaben niederschlage. Dennoch sei diese europäische Entwicklung schon sehr früh und intensiv verfolgt worden. Dies gelte im Übrigen auch für die gesamte Entwicklung in der „Gesundheitswirtschaft": Es gehe dabei ja nicht nur um die gute Versorgung der Versicherten in Deutschland, sondern auch um ein konkurrenzfähiges Angebot an gesundheitlicher Infrastruktur für Interessenten/Patienten aus dem Ausland. Gesundheitspolitik sei nicht zuletzt heute auch Standortpolitik.

\section{Regionale Mitverantwortung für gute europä- ische Gesundheitspolitik}

In ihrem Überblick zur Kompetenzverteilung in der europäischen Gesundheitspolitik bezog sich Ulla Kalbfleisch-Kottsieper zum Teil auf das, was Borowsky bereits in seinem Vortrag zum Verfassungsentwurf festgestellt hatte. Zusätzlich verwies sie aber auf die interessanten Stellungnahmen zu diesem Bereich im Ausschuss der Regionen, im Kongress der Regionen und Kommunen beim Europarat und der Versammlung der Regionen Europas. Besonders in der grenzüberschreitenden $\mathrm{Zu}$ sammenarbeit im Rahmen der Gesundheitspolitik bewähre sich das, was die Regionen und Kommunen seit vielen Jahren in verschiedensten Politikbereichen an vertrauensbildenden Maßnahmen und konkreter Projektzusammenarbeit entwickelt hätten. Nach Meinung von Kalbfleisch-Kottsieper wäre es schade, wenn dem im Reformvertrag nicht Rechnung getragen werden würde. ${ }^{4}$

Auch wenn die Gesundheitsministerkonferenz der deutschen Länder ${ }^{5}$ nochmals auf ihre bekannten Positionen hingewiesen habe, dass neben der Ausklammerung der Gesundheits- dienstleistungen aus der europäischen Dienstleistungsrichtlinie auch künftig die alleinige Zuständigkeit der Mitgliedstaaten für die Ausgestaltung und Finanzierung ihres nationalen Gesundheitssystems und deren Prinzipien zu wahren sei, stellte Kalbfleisch-Kottsieper dennoch in diesem Hort des föderalen Beharrens auch eine gewisse Bewegung fest. Im Beschluss der Ministerkonferenz heißt es unter anderem, man sähe auf europäischer Ebene in Bezug auf Gesundheitsdienstleistungen ,einen Bedarf" für die Schaffung von Rechtssicherheit. Dies gelte sowohl für die Definition der Gesundheitsdienstleistungen und für deren Kostenerstattung. Für eine generelle, gemeinschaftliche Regelung zur grenzüberschreitenden Gesundheitsversorgung bestehe nach Ansicht der Gesundheitsminister der Länder aber „kein aktueller Bedarf“. Dies gelte ebenso für die Absicht der Europäischen Kommission, für bestehende Netzwerke von Referenzzentren einen einheitlichen rechtlichen Rahmen zu schaffen. Dies werde - gemeinsam mit dem Bund - abgelehnt, obwohl der dort stattfindende Erfahrungsaustausch durchaus einen europäischen Mehrwert darstelle und im Wege bilateraler Verträge auch verstärkt genutzt werden sollte. Der generelle gesundheitspolitische Ansatz der Europäischen Kommission für eine stärkere Berücksichtigung des Gesundheitsaspektes in allen sonstigen Politikbereichen finde Unterstützung. Es solle aber hierfür eine Prioritätenliste erstellt und im Sinne des Subsidiaritätsprinzips bei deren Umsetzung die bestehenden Einrichtungen der Mitgliedstaaten genutzt werden, um den Aufbau unnötiger neuer Strukturen zu vermeiden.

Fazit: EU-Gesundheitspolitik wird sich dynamisieren, trotz - oder gerade auch wegen der, Verweigerung ' von Kompetenztransfer

In der Schlussdiskussion wurde nochmals deutlich, dass ein Widerspruch zwischen dem

4 Im Vertrag von Lissabon sind die Neuformulierungen aus dem VVE zur grenzüberschreitenden Zusammenarbeit im Fall schwerwiegender Gesundheitsgefahren und zur Verbesserung der Komplementarität der Gesundheitsdienste im Grenzgebiet weiter enthalten.

5 Sonderkonferenz der Gesundheitsminister im Rahmen der deutschen EU-Ratspräsidentschaft, Stuttgart, 8. März 2007. 
Beharrungsvermögen nationaler Politikverantwortlicher und den eigentlichen Interessen bereits sehr viel europäischer denkender Patienten und auch vieler junger Ärzte besteht. Patienten orientieren sich am Preis und an der Verfügbarkeit des Produktes ,Gesundheit'. Junge Ärzte gehen - Erasmuserfahren - ins europäische Ausland, wenn Arbeitsbedingungen und Bezahlung in Deutschland nicht den Erwartungen entsprechen. Selbst die Krankenkassen als einer der wichtigsten Akteure im gesundheitspolitischen Machtpoker europäisieren ihre Angebote. Wenn die deutsche Europapolitik nicht nach dem Muster politics follows facts verfahren will, wird sie sich in der europäischen Realität aktiver einbringen müssen. Die Umsetzung des Nichtraucherschutzes und auch das Interesse und die Akzeptanz, die andere europäische Initiativen, zum Beispiel im Kampf gegen den Krebs und Alkoholmissbrauch bei den europäischen Bürgern - und bei den Medien - gefunden haben und finden werden, machen deutlich, dass die Europäische Union Politik auch ohne umfassende Kompetenzen durchsetzen kann, wenn sie die richtigen Themen mit nachhaltigem politischen Druck konsequent benennt und Schritt für Schritt umsetzt. Eine solche Politik nützt den Menschen in Europa und sollte deshalb auch von den Regierungen der Mitgliedstaaten mitgetragen werden.

\section{Föderalismus in Deutschland und im europäischen Vergleich}

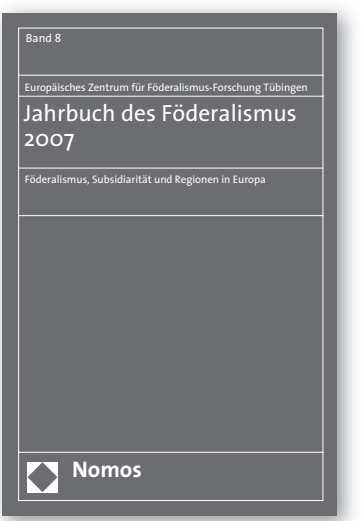

Jahrbuch des Föderalismus 2007

Föderalismus, Subsidiarität und Regionen in Europa

Vom Europäischen Zentrum für Föderalismus-

Forschung Tübingen

2008, 670 S., geb., 76,-€, ISBN 978-3-8329-2961-9

Die achte Ausgabe des Jahrbuch behandelt in 42 Einzelbeiträgen das breite Spektrum von Fragen der Territorialstruktur im heutigen Europa: insbesondere die Föderalismus-Reform in Deutschland, Regionalisierungsprozesse in zahlreichen europäischen Staaten, die künftige Ordnung der EU, die Rolle von Regionen und die Geltung des Subsidiaritätsprinzips.

Bitte bestellen Sie bei Ihrer Buchhandlung oder bei Nomos | Telefon 07221/2104-37 | Fax -43 www.nomos.de | sabine.horn@nomos.de 